\title{
Measuring Bitrate and Quality Trade-off in a Fast Region-of-Interest Based Video Coding
}

\author{
Salahuddin Azad, Wei Song, and Dian Tjondronegoro \\ Faculty of Science and Technology, \\ Queensland University of Technology, Brisbane 4001, Australia \\ salahuddinalazad@yahoo.com \\ $\{$ w1.song, dian\}@qut.edu.au
}

\begin{abstract}
Prevailing video adaptation solutions change the quality of the video uniformly throughout the whole frame in the bitrate adjustment process; while region-of-interest (ROI)-based solutions selectively retains the quality in the areas of the frame where the viewers are more likely to pay more attention to. ROI-based coding can improve perceptual quality and viewer satisfaction while trading off some bandwidth. However, there has been no comprehensive study to measure the bitrate vs. perceptual quality trade-off so far. The paper proposes an ROI detection scheme for videos, which is characterized with low computational complexity and robustness, and measures the bitrate vs. quality trade-off for ROI-based encoding using a state-of-the-art H.264/AVC encoder to justify the viability of this type of encoding method. The results from the subjective quality test reveal that ROI-based encoding achieves a significant perceptual quality improvement over the encoding with uniform quality at the cost of slightly more bits. Based on the bitrate measurements and subjective quality assessments, the bitrate and the perceptual quality estimation models for non-scalable ROI-based video coding (AVC) are developed, which are found to be similar to the models for scalable video coding (SVC).
\end{abstract}

Key words: Bitrate modeling, Quality modeling, Region-of-Interest, H.264.

\section{Introduction}

Adaptive video streaming adjusts the bitrate of the video stream and the perceptual quality to meet the current network bandwidth constraint. Existing adaptation solutions affect the quality of the video equally throughout the whole frame in the bitrate adjustment process. However, it has been found that there are certain regions in the video frame where the viewers mostly concentrate on than other regions [10]. This is due to the highly non-uniform distribution of photoreceptors on the retina in human eyes. In the retina, only a small region of 2-5 degrees of visual angle (the fovea) around the center of gaze is captured at high resolution, with logarithmic resolution falloff with the distance from the center [4]. Thus, it may not be useful to encode each video frame with uniform 
quality, since the human observers will crisply perceive only a very small fraction of each frame, depending on their current point of fixation. Region-of-interest (ROI)-based video coding can improve the apparent perceptual quality of videos by selectively retaining the quality in the areas where the viewers are more likely to pay more attention to [5].

During the last three decades, a great deal of object detection or visual attention models have been developed. The techniques consider the human visual features and are able to be used to detect the regions of interest. For example, motion vector-based object detection[2] is a faster and robust approach and can be implemented in near real-time and compression domain. However, it is prone to global motion. An segmentation and region growing method [7]is based on color-texture features and tracks the segmented objects. The skincolor \& face detection mehtods[15][16] detect the important area human face by extracting skin-color pixels. The saliency maps method[11]integrates different visual features (color, orientation, movement etc.) into one single topographic saliency map and select spotlight of attention. A recent method is to combine various features and color contrast, object motion and face detection to determine the ROIs[1]. However, these solutions are computationally expensive and time consuming, which makes unsuitable for real-time processing of large number of videos. Although it is expected that higher accuracy of ROI detection can be achieved through using more features of video content, it is highly difficult to create a proper detection algorithm with various features to suit different videos. In fact, according to our latest study on evaluation of objective ROI detection methods with subjective assessment, using motion feature only gains a similar accuracy in most types of video contents.

The usual method for ROI-based video coding is to use lower quantization parameter (QP) for the macroblocks within the ROI and higher QP for the macroblocks outside the ROI so that it can achieve higher perceptual quality than the uniform quality encoding. In trade-off, ROI-based encoding consumes more bandwidth than uniform encoding. However, ROI-based encoding still takes significantly less bandwidth than the maximum quality video as it degrades quality in most of the areas. In a nutshell, ROI-based coding should be the winner on both sides - retaining quality and saving bitrate. To the best of the authors' knowledge, there has been no comprehensive study so far to measure the cost-performance trade-off for ROI-based encoding.

The recent objective assessment [6] on ROI-based encoding has analyzed the impact of two QP degradation methods - linear quality distance adaptation and logarithmic quality distance adaptation on the human visual system. The former method degrades the quality of each macroblock linearly with distance from the area of maximum user interest (MAUI), while later method degrades the quality of the macroblock logarithmically with distance from MAUI. The experimental results suggest that if the viewer is highly interested in certain areas and have a very little interest in other areas, the linear method performs better. However, if the user has relatively balanced interest in various areas of the image with an obvious peak in MAUI, the logarithmic method performs 
better. Nevertheless, both the schemes have the disadvantage of providing very poor local quality at the furthest points from the MAUI. This is the reason why the paper considers only two distinct qualities for the ROI and non-ROI areas. The bitrate and perceptual quality modeling of video bitstreams allows a video adaptation system to estimate the bitrate and perceptual quality of degraded bitstreams without actually extracting them and this is particularly useful when the adaptation decision is taken at the client-side. The reduction in bitrate for scalable video bitstreams due to adjustment in encoding parameters - frame rate, quantization parameter and spatial resolution has been modeled in [3] and [18]. The modeling of bitrate in terms of QP for non-scalable MPEG video bitstreams has been developed in [8]. The modeling of bitrate for ROI-based encoding is yet not studied, which is really necessary to design a ROI-based video adaptation system. The perceptual quality modeling of scalable video bitstreams was developed in [17] and [14]. Both the bitrate and perceptual quality models contain parameters that are somehow dependent on the actual contents of the videos. The exact relationship between these parameters and the video contents is hard to determine accurately and still a challenging research topic.

The main objective of this paper is to investigate the cost-performance benefit of ROI-based quality adjustment with non-scalable H.264/MPEG-4 AVC encoding [17]. The paper uses the motion-based ROI detection scheme to automatically identify the ROIs in the test videos. A number of standard and nonstandard videos were encoded with different quantization parameters with both uniform quality and ROI-based quality. The analysis shows that the additional bitrate for enhancing the quality of ROI is on average $10 \%$ of the original bitrate without ROI. A subjective quality test was also conducted on the encoded videos to see the perceptual quality gain compared to the uniform quality encoding. The test results show that the quality improvement of ROI-based coding over uniform encoding is on average $10 \%$. Besides drawing the above conclusion, the paper develops the bitrate and quality models for non-scalable ROI-based encoding that are found be similar to models for scalable videos [18].

The rest of the paper is organized as follows. Section 2 addresses the motionbased ROI detection scheme and Section 3 describes the experimental setup for bitrate and quality measurement for ROI-based encoding of videos. The analysis of the bitrate and perceptual quality of ROI-based encoding and comparison with the uniform quality encoding are presented in Section 4 and 5 respectively. Section 6 provides a further discussion. Finally, Section 7 concludes the paper.

\section{Motion-based ROI Detection}

The motion-based ROI detection scheme used in this paper to produce ROIbased encoding is done using $\mathrm{YUV}$ ( $\mathrm{YCbCr}$ ) color space. The technique is based on the fact that the most important objects (e.g., the anchor in the news videos) are most likely to remain around the central region in the frame throughout an entire scene. The approach divides each video into a number of shots based on the number of pixels changed between successive frames. In the next step, the 
statistics for likelihood of the luma changes throughout all frames in an entire scene are calculated and the position of the fixed size rectangular region where the change is most likely is determined. The center of that region is considered as the center of ROI. The proposed technique consists of the following steps:

1. Scene Change Detection

The scene change is simply determined by whether the changed luminous pixels in each frame exceeds a threshold value. The threshold value is defined to set the minimum distance between two successive scenes.

2. Maximum Change Location Detection

The next step finds the location of the rectangular region where the maximum number pixel change occurs in each frame. The operation traverses the whole frame with a certain stepsize at both horizontal and vertical directions. Using the statistics of all frames throughout a given scene, a histogram is created which records the frequency of occurrences of the maximum number of pixel changes within each rectangular area. This step is executed on each frame of the video.

3. Forming Cluster of Rectangular Areas

This step forms the cluster of rectangular areas in the previous step. In this step, a greater rectangular area represents the cluster which contains a central rectangular area and all its surrounding and overlapping rectangular areas. The clusters centers are separated by $\Delta$ distance apart. The value of $\Delta$ is chosen such that clusters formed this way overlap with their neighboring clusters. A second level histogram is then constructed that actually records the total frequency of the occurrences of maximum number of pixel changes within that particular cluster throughout an entire scene. This step is carried out once in each scene.

4. Finding the Center of ROI

The center of ROI is the cluster with the maximum value of the frequency. Given the center of the ROI, it is possible to choose an arbitrary sized and shaped ROI around that center.

The outputs of the proposed ROI detection algorithm carried out on six news videos ("GMnews", "Disease", "AFL", "Tennis") are shown in Fig. 1.In the experiment, the ROI is a rectangular area having the width and height half of that of the original frame.Unlike the existing motion-based algorithm[2] for detecting ROIs in video frames, the novelty of the proposed scheme is that it can detect the degree of importance of the objects using the extent and clustering of luma changes.

\section{Experiment Setup}

The experiment used eight standard videos ("city", "crew", "football", "foreman", "harbour", "mobile", "news", "soccer") with CIF resolution and six news videos ("AFL", "CQfire", "Disease", "GMnews", "Spiderman", "Tennis") with 480x360 resolution. Each video was first encoded with uniform quality with four 

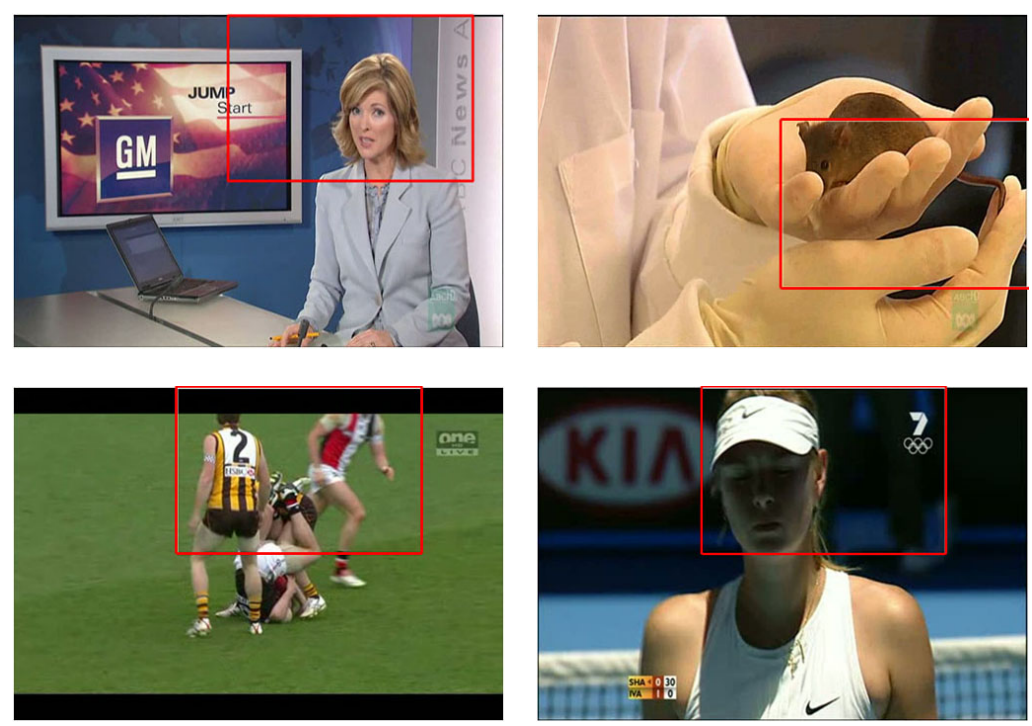

Fig. 1. The ROIs (inside the red color rectangles) detected by the proposed scheme in (a) "GMnews" (b) "Disease" (c) "AFL" and (d) "Tennis" news videos.

different QP values $28,32,36,40$ which correspond to quantization stepsize values $q=16,26,40,64$ respectively. Later on, the same videos were encoded with ROI-based quality with the same series of QP values for areas outside ROI but keeping the QP within ROI fixed at 28 (corresponding to $q_{\text {min }}=16$ ). The encoding of the videos was done with a custom-modified x264 encoder, which can encode a frame with uniform quality or different quality for ROI and nonROI areas (x264 is a state-of-the-art free encoder library which produces video bitstream in H.264/MPEG-4 AVC format [17]. The ROI for each video is a rectangular area having the height and the width equal to a half the height and width of the original frame respectively. The area of ROI is a quarter of the area of the original frame, and according to the authors' experiments, this size is reasonably optimal. This is because for a too large ROI, the bitrate will be higher, whereas for a too small a ROI, it may not fully cover the objects of interest and as a result, the perceptual quality improvement may not be noticeable by the users.

For the subjective quality test, a subset of the aforementioned standard videos ("city", "crew", "football", "foreman", "harbour", "mobile", "news", "soccer") and news videos ("AFL", "Disease", "GMnews", "Tennis") as test materials. Subjective test was performed in a laboratory, which is a soundproof meeting room with controlled lighting conditions according to the ITU's recommendations [13]. The test device was a SAMSUNG R700 laptop with a 17inch TFT LCD monitor, which display resolution was set as 1280x768. A total of 20 viewers took part in the subjective perceptual video quality assessment. 
Among the participants, there were 12 males and 8 females, ten of them with image/video processing experience. The age of the participants ranged from 22 to 36 and all of them reported to have normal vision. Each test content was presented with the following conditions: an explicit reference and a hidden reference (high uniform quality with $\mathrm{QP}=28$ ) and six impaired test sequences (uniform quality and ROI-based quality at $\mathrm{QP}=32,36,40$ ). After watching each test sequence, the subject used a 11-scale (0-10) slider to mark the impaired quality of the watched video to the reference video. After receiving all scores from the subjects, the normality of data distribution was examined by 1-Kolmogorov-Smirnov (K-S) test $(p>.05)$ [9]. The mean opinion score (MOS) for each sequence was calculated by averaging all scores for that sequence.

\section{Bitrate Modeling}

Fig. $2 \mathrm{a}$ and $2 \mathrm{~b}$ show the normalized bitrates due to the increment in quantization stepsize using uniform quality encoding for standard and news videos respectively, while Fig. $3 \mathrm{a}$ and $3 \mathrm{~b}$ show the same using ROI-based encoding. The normalized bitrate means the ratio of the actual bitrate of a given bitstream to the bitrate of the maximum quality (corresponding to $q_{\min }=16$ ) bitstream. Based on the normalized bitrate curves for the uniform quality encoding, the normalized bitrate can be modeled as an inverse power function, i.e.,

$$
R(q)=\left(\frac{q}{q_{\min }}\right)^{-a} \quad a>1
$$

considering the minimum quantization stepsize as $q_{\min }=16$. From the curve fitting data, it can be observed that the value of a slightly varies for different standard videos, while the value of $a$ is uniform for the news videos. The reason behind the deviation is that the range of quantization parameters chosen was small compared to [19]. The average value of $a$ is found to be 1.2 approximately, which is the same as the one reported in [19], despite the fact that the later model was proposed for scalable videos. For ROI-based encoding, the quantization stepsize is fixed at $q_{\min }=16$ within the ROI which is one quarter of the frame. Hence the non-ROI area, which is the remaining three quarters of the frame, accounts for the bitrate reduction. Therefore, the normalized bitrate for ROI-based encoding can be model as

$$
R_{c}(q)=\alpha+(1-\alpha)\left(\frac{q}{q_{\min }}\right)^{-a} \quad a>1
$$

According to the ROI size considered in the paper ( $1 / 4$ of the whole frame), Fig. 3a and 3b confirm that the bitrate curves for the news videos are consistent with the model in formula (2), while curves for the news videos deviate slightly from the model. Specially, videos of "city", "foreman", "harbour", "mobile" achieve better compression than the model in (2) mainly due to their uniform motions. The reason why news videos achieve more consistent curves than standard videos is that each of the standard videos contains only a single shot and 
the contents vary largely from one video to another; in contrast, each of the news videos contains multiple and diverse shots and the length is twice as that of a standard video. Fig. $4 \mathrm{a}$ and $4 \mathrm{~b}$ show the differences between the normalized bitrates of the uniform quality and ROI-based encodings for standard and news videos respectively. As using $q_{\min }=16$ into the ROI, the difference between the two methods is zero when $q=16$. In other cases, the additional bitrates for ROIbased encodings exceed uniform quality encoding range from $6 \%$ to $23 \%$ with a median of $10 \%$ for news videos. On the other hand, Fig. 5a and 5b show the differences between the normalized bitrates of the maximum quality encoding (using $\mathrm{q}=16$ for the whole frame) and ROI-based encodings for standard and news videos respectively. From these figures, the bitrates for ROI-based encodings are far less than the bitrate for the maximum quality, the bitrate differences ranging from $40 \%$ to $80 \%$ with a median of $60 \%$.

\section{Quality Modeling}

Fig. $6 \mathrm{a}$ and $6 \mathrm{~b}$ show the normalized MOS due to the increment in quantization stepsize using uniform quality encoding for standard and news videos respectively, and Fig. $7 \mathrm{a}$ and $7 \mathrm{~b}$ show the same for ROI-based encoding. The normalized MOS means the ratio of the actual MOS for a given bitstream to the MOS for the maximum quality (corresponding to $q_{\min }=16$ ) bitstream. The normalized MOS curves for standard videos are not much consistent, while the curves for news videos are. This is because the response of the viewers to the changes in quality largely depends on the content of the video. Since the content of a standard video remains almost the same throughout the playback period and the content widely differs from each another, the user's perception of quality also varies accordingly. Based on the normalized MOS curves in Fig. 6a and $6 \mathrm{~b}$, the normalized perceptual quality can be modeled as a falling exponential function, i.e.,

$$
Q(q)=e^{-c} e^{-c\left(\frac{q}{q_{\min }}\right)} \quad c<1
$$

where the minimum quantization stepsize as $q_{\min }=16$. Based on the curve fitting data, the approximate value of $c$ is found to be 0.35 which is larger than the one reported in [19]. Although this is a negative exponential function, the small value of $c$ makes drop in normalized MOS much slower than the drop in normalized bitrate. If the normalized MOS is assumed to follow the same trend as the normalized bitrate for ROI-based encoding, the normalized perceptual quality for ROI-encoded videos can be expressed as

$$
Q_{c}(q)=\alpha+(1-\alpha) e^{-c} e^{-c\left(\frac{q}{q_{m i n}}\right)} \quad c<1
$$

Fig. 7b confirm that normalized perceptual quality for ROI-based encodings is generally very close to $Q_{c}(q)$. Fig. $8 \mathrm{a}$ and $8 \mathrm{~b}$ show the differences between normalized MOSs of the uniform quality and ROI-based encodings bitrate for standard and news videos respectively. According to these figures, the quality improvements in ROI-based encodings over uniform encodings range from $5 \%$ 
to $20 \%$ with a median of $10 \%$. On the other hand, Fig. 9a and 9b show the differences between the normalized MOSs of the maximum quality encodings and ROI-based encodings for standard and news videos respectively. According to these figures, the quality difference between the ROI-based coding and maximum quality video ranges from $10 \%$ to $70 \%$ with a median of $40 \%$.

\section{Discussion}

Careful observation reveals that the lowest normalized bitrate achieved in the experiment is around $20 \%$ of the maximum bitrate, whereas the lowest perceptual quality is $40 \%$ of the maximum quality. Comparing the bitrate overhead and quality improvement between ROI-based encoding and uniform quality encoding, it can be concluded that the median of $10 \%$ for quality improvement significantly outweighs the median of $10 \%$ bitrate overhead as the normalized quality curve drops much slower than the bitrate curve. Also, the small bitrate overhead for ROI means that it can be a useful tool for fine-tuning bitrates.

In spite of the general benefit from ROI-based video coding, it should be noted that it does not contribute to all content types. From Fig. 8a and 8b, it can be observed that ROI-based encoding cannot guarantee quality improvement for shots ("AFL", "football", "city" and "mobile") with global motions and/or too many objects. This is ascribed to two main reasons: content feature and ROI detection. On the one hand, for videos with high textual complexity and slow global motion (e.g., "mobile" and "city"), their perceptual quality is not very impacted by quantization level(see slowly downed curves in Fig.6a and Fig.7a). On the other hand, the automatic ROI detection scheme with fixed size ROI used in this paper, may not work perfectly for the videos with many objects (e.g., "football" and "AFL") and/or global motions. Therefore, we argue that encoding this type of shots, uniform encoding rather than ROI-based encoding will be the better choice. Future ROI detection schemes should be able to take care of this kind of shots.

\section{Conclusion}

The paper measures the bitrate vs. perceptual quality trade-off for non-scalable ROI-based encoding using H.264 encoder. Based on the quantitative measurements, a bitrate model and a perceptual quality model are developed to predict the bitrate and perceptual quality of the ROI-based encoding so that the adaptation decision can be easily made at the client-side. The experiment shows that ROI-based encoding achieved more perceptual quality than the bandwidth traded off. Moreover, it is observed that the quality gain is influenced by content features, quantization stepsize, and the efficacy of ROI detection. Development of more effective ROI detection schemes will improve the perceptual quality even further. In addition, since only one ROI and fixed ROI size were used in this paper, the impacts of multiple regions of interest and different ROI size should be addressed in the future work. 

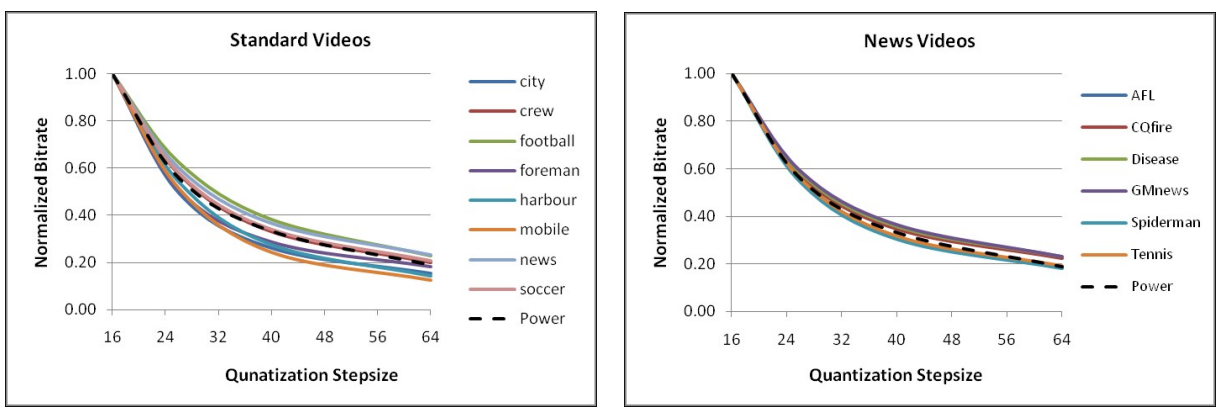

Fig. 2. Normalized bitrate for (a) standard and (b) news videos for different quantization stepsizes compressed with uniform quality encoding.
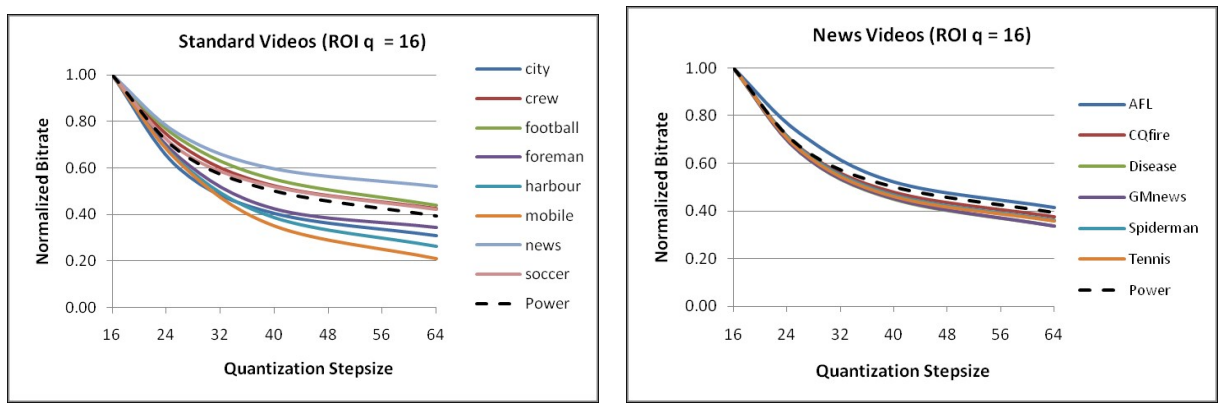

Fig. 3. Normalized bitrate for (a) standard and (b) news videos for different quantization stepsizes compressed with ROI-based encoding when $q_{\min }=16$ inside ROI.
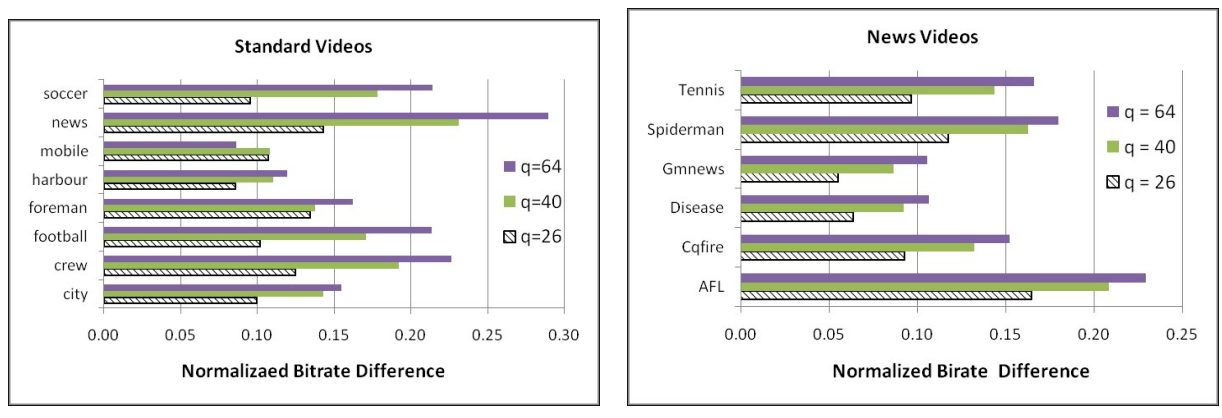

Fig. 4. Pair-wise normalized bitrate differences under given $q$ values between ROIbased encodings with $q_{\min }=16$ inside ROI and the uniform quality encoding for (a) standard and (b) news videos.
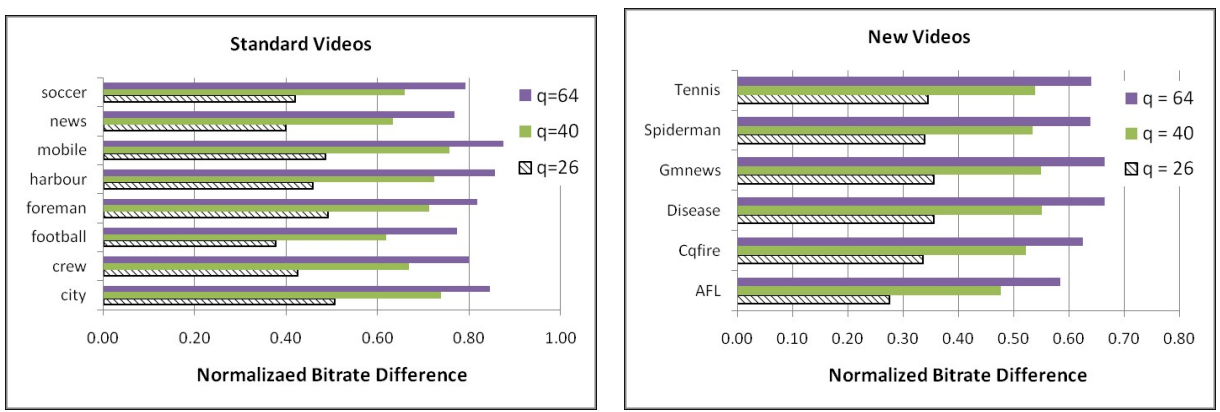

Fig. 5. Pair-wise normalized bitrate differences between the maximum quality encoding and ROI-based encodings with $q_{\min }=16$ inside ROI and given $q$ values outside ROI for (a) standard and (b) news videos. 

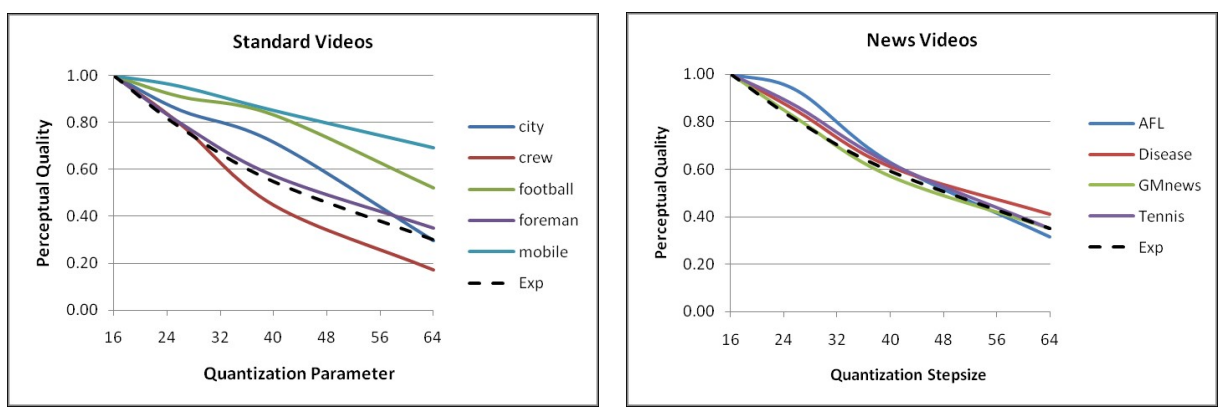

Fig. 6. Normalized perceptual quality for (a) standard and (b) news videos for different quantization stepsizes compressed with uniform quality encoding.
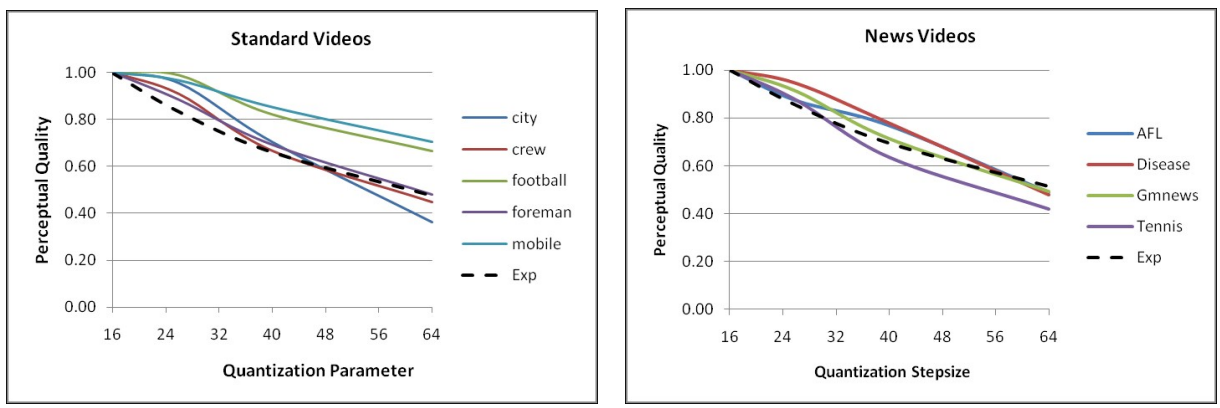

Fig. 7. Normalized perceptual quality for (a) standard and (b) news videos for different quantization stepsizes compressed with ROI-based encoding when $q_{\min }=16$ inside ROI.
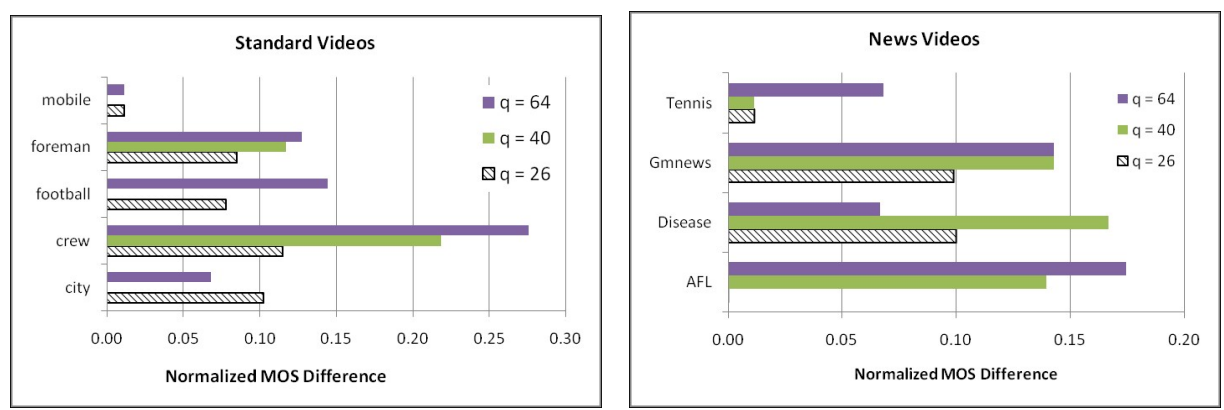

Fig. 8. Pair-wise normalized MOS differences under given $q$ values between ROI-based encodings with $q_{\min }=16$ inside ROI and the uniform quality encoding for (a) standard and (b) news videos.
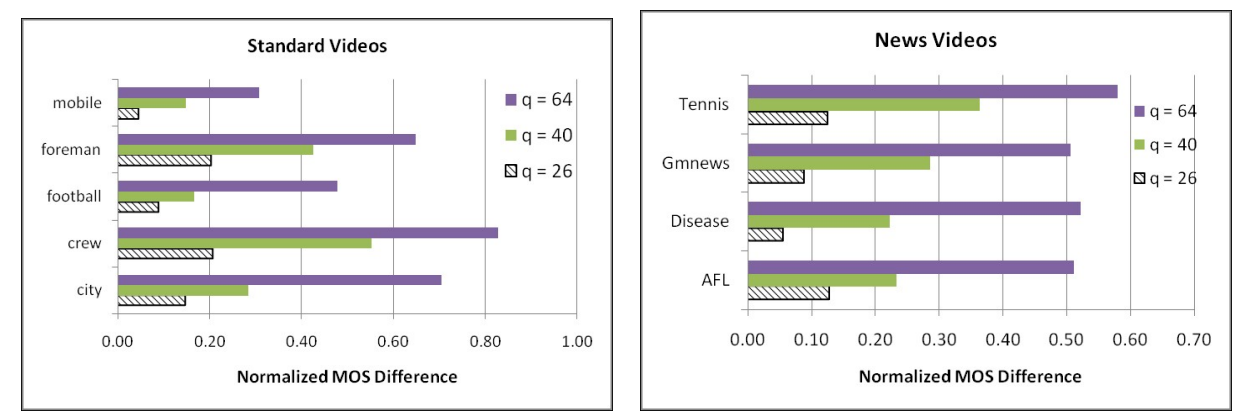

Fig. 9. Pair-wise normalized MOS differences between the maximum quality encoding and ROI-based encodings with $q_{\min }=16$ inside ROI and given $q$ values outside ROI for (a) standard and (b) news videos. 


\section{References}

1. Abdollahian, G., Taskiran, C.M., Pizlo, Z., and Delp, E.J.:Camera Motion-based Analysis of User Generated Video. IEEE Trans. on Multimedia, 12,1, pp. 28-41. IEEE Press (2010)

2. Ahmad, A.M.A.: Content-based Video Streaming Approaches and Challenges. In: Ibrahim, I.K. (ed.): Handbook of Research on Mobile Multimedia. Idea group Reference, London 357-367 (2006)

3. Azad, S., Song, W., and Tjondronegoro, D.: Bitrate Modeling of Scalable Videos Using Quantization Parameter, Frame rate and Spatial Resolution. In Proc. of ICASSP'10. pp. 2334-2337. IEEE Press (2010)

4. Brian A. Wandell.: Foundations of Vision. Sunderland, MA: Sinauer (1995)

5. Chi, M., Chen, M., Yeh, C., and Jhu, J.: Region-of-Interest Video Coding Based on Rate and Distortion Variations for H.263+. Image Commun. Vol.23, 2, pp. 127-142 (2008).

6. Ciubotaro, B., Muntean, G-M., and Ghinea, G.: Objective Assessment of Region of Interest-aware Adaptive Multimedia Streaming Quality. IEEE Trans. on Broadcasting. Vol.55,2, pp.202-212 (1982)

7. Deng, Y., and Manjunath, B.S.: Unsupervised Segmentation of Color-Texture Regions in Images and Video, IEEE Trans. on Pattern Analysis and Machine Intelligence, Vol.23,8, pp.800-810 (2001)

8. Ding, W., and Lu, B.: Rate Control of MPEG Video Coding and Recording by Rate Quantization Modeling. IEEE Trans. on Circuits and Sys. for Video Technology, 6, pp.12-20 (1996)

9. Eadie, W.T., Drijard, D., James, F.E., Roos, M., and Sadoulet, B.: Statistical Methods in Experimental Physics. Amsterdam: North-Holland. pp.269-271 (1971)

10. Gulliver, S., and Ghinea, G.: Stars in Their Eyes: What Eye Tracking Reveals about Multimedia Perceptual Quality. IEEE Trans. on Sys., Man and Cybernetics, Vol.34,4, pp.472-482 (2004)

11. Guo, C., and Zhang, L.: A novel Multiresolution Spatiotemporal Saliency Detection Model and Its Applications in Image and Video Compression. IEEE Trans. on Image Processing. Vol.19,1, pp.185-198 (2010)

12. X264 codec, http://www.videolan.org/developers/x264.html

13. ITU-T: Subjective video quality assessment methods for multimedia applications. P.910 Recommendation (1999)

14. Ou, Y.-F., Ma, Z., and Wang, Y.: A novel quality metric for compressed video considering both frame rate and quantization artefacts. In Proc.of Intl. Workshop Video Processing and Quality Metrics for Consumer (VPQM).(2009)

15. Peer, P., and Solina, F.: An automatic human face detection method, in Proc. of CVWW'99, pp.122-130 (1999)

16. Solina, F., Peer, P., Batagelj, B., and Juvan, Samo: 15 seconds of fame - an interactive computer vision-based art installation. in Proc. of ICARCV 2002, pp.198-204 (2002)

17. Sullivan, G.J., Topiwala, P. and Luthra, A.: The H.264/AVC Advanced Video Coding Standard: Overview and Introduction to the Fidelity Range Extensions. In Pro. of the SPIE Conf. on Applications of Digital Image Processing pp.1-22(2004)

18. Sullivan, G.J., Wiegand, T., and Schwarz, H.: Amd.3 Scalable video coding, ISO/IEC JTC1/SC29/WG11, MPEG08/N9574, Antalya, TR. (2008)

19. Wang, Y., Ma, Z., and Ou, Y.-F.: Modeling rate and perceptual quality of scalable videos as functions of quantization and frame rate and its application in scalable video adaptation. In Proc. of 7th International Packet Video Workshop. (2009) 